\title{
Editorial
}

\section{Availability Management in Mediated Communication}

In recent decades, communication media have made strides in enabling efficient and effective communication and collaboration. An increasing range of media are available providing a lot of the richness of human-human communication when this is mediated. The increased availability of such media communication media and the increasingly continuous connectivity of individuals can have fundamental impact on the way we work together with colleagues, or the way we socialize with friends and family. Individuals can initiate contacts opportunistically and with great ease, which at the receiving end creates the problem of managing one's availability for communication [1-3]. This need is manifested at work, where one has to avoid interrupting or being interrupted for the sake of productivity, reduced stress, and even for avoiding erroneous action; it is also manifested at leisure when individuals need to secure moments of leisure, relaxation, or private enjoyment alone or with family and friends [4-6].

The subject of availability and interruptions management has been broadly discussed in the fields of Human Computer Interaction and its subfield of Computer Supported Cooperative Work [7-11]. Although some efforts have been made to enable people to deal with multiple streams of communication, the actual problem of availability management over communication media remains unsolved and the ways in which our future workplace and our social life will be impacted are still effervescent fields of research.

It has become clear that a purely technologically-oriented approach to resolve these challenges issue is insufficient. Technology on its own seems to be unable to mimic the complexity of human communicative choices [6,12]. A new approach emerges that aims to inform the design of communication media leveraging on extant social skills of people to manage communications $[5,13]$.

The articles in this special issue contribute in the body of knowledge related to this approach. Through a number of experimental and empirical studies the meaning of availability and communication management is revisited. Different user requirements pertaining to their informational and social needs are discussed and a new directions for future research are presented.

Curtis and Janssen study challenges and opportunities for supporting one's ability to stay connected and up-to- date with colleagues at work. Their study focuses on the communication needs of mobile workers examining the constructs of staying 'in-sync' and 'in-touch' with colleagues or in other words to be up to date and to feel connected to them. They particularly focus on determining the requirements of one subgroup of mobile workers: Traditionalists for whom a transition from predominately co-located interactions to those technologically-mediated seems to be most difficult. They suggest a number of technological improvements that could effectively support these people in their attempts to stay in sync and in touch with colleagues.

Arora, Gonzalez and Payne present a study aiming to understand work fragmentation and multitasking caused by mediated communication tools like e-mail and phone. They analyze work-related and non-work-related sources of interruption and inspect how this distinction relates to the particular type of communication technology and the characteristics of the interrupter. Their results provide a better understanding regarding different social functions served by informal communication at the workplace and their impact on effective collaboration.

Szostek, Markopoulos, and Eggen report three studies exploring the nature of availability and examining the impact different factors have on that status. They note that communications requiring little time that are important and urgent have a higher chance to be immediately handled regardless of the communication channel they are being held with. They also observe that social proximity has a great positive influence of an interruption outcome in face-to-face communications but it appears to be an insufficient predictor for email communications. Their results suggest the dynamic nature of availability which is collaboratively negotiated by the communicators and depends on factors such as social proximity, nature of the interruption subject and anticipated interruption duration.

Ruyter, Baha, Pijl and Markopoulos report two experiments evaluating how empathic skills influence one's ability to assess another person's availability in a home environment by comparing full video image and a silhouette based obfuscation. The results indicate that empathy is important in making availability judgments based on limited information such as provided in a silhouette video visualization. Their findings motivate a design approach that aims to enable people to apply empathetic and social cognition skills when making availability judgments.

\section{REFERENCES}

[1] Czerwinski M, Horvitz E, Wilhite S. A diary study of task switching and interruptions: Proceedings of the SIGCHI conference on Human factors in computing systems; April 24-29; 2004. Vienna Austria. 
[2] Gonzalez VM, Mark G. Constant, constant, multi-tasking craziness: managing multiple working spheres. Proceedings of the SIGCHI conference on Human factors in computing systems; April 24-29; 2004; Vienna Austria.

[3] Gonzalez VM, Mark G. Managing currents of work: Multi-tasking among multiple collaborations. Proceedings of ECSCW European conference on computer supported cooperative work 2005; September 18-22, 2005; Paris, France.

[4] Kraut RE, Fish RS, Root RW, Chalfonte BL. Informal communication in organizations: Form, function, and technology. Human reactions to technology. In Proceedings of claremont symposium on applied social psychology 1990.

[5] Szostek Matysiak A, Karapanos E, Eggen B, Holenderski M. Understanding the implications of social translucence for systems supporting communication at work. Proceedings of the ACM conference on computer supported cooperative work 2008; San Diego California, USA.

[6] Patil S, Lai J. Who gets to know what when: configuring privacy permissions in an awareness application. In Proceedings of the SIGCHI conference on Human factors in computing systems: April 2-7, 2005; Portland, USA.

[7] Begole B, Matsakis NE, Tang JC. Lilsys: Sensing una vailability. CSCW 2004; 1: 511-4.

[8] Dabbish L, Kraut R. Controlling interruptions: Awareness displays and social motivation for coordination. CSCW 2004; 1: 182-91.

[9] Fogarty J, Hudson SE, Atkeson, CG, et al. Predicting human interruptability with sensors. ACM Trans Comput Hum Interact 2005; 12(1):11946.

[10] Romero NA, Szostek Matysiak A, Kaptein M, Markopoulos P. Behaviors and preferences when coordinating mediated interruptions: Social and system influence. In Proceedings ECSCW European conference on computer supported cooperative work 2007, Limerick, Ireland.

[11] Whittaker S, Frohlich D, Daly-Jones O. Informal workplace communication: What is it like and how might we support it? Proceedings of the SIGCHI conference on Human factors in computing systems 1994; Boston, Massachusetts, USA

[12] Romero NA, Markopoulos P. Grounding interpersonal privacy in mediated settings. In Proceedings of the ACM 2009 international conference on supporting group work May 10-13, 2009; Sanibel Island, Florida, USA.

[13] Erickson T, Kellogg WA. Social translucence: An approach to designing systems that support social processes. ACM Trans Comput Hum Interact 2000; 7(1): 59-83.

\section{Agnieszka Szostek (Co-Guest Editor)}

Institute of Information Processing

Al. Niepodległości $188 \mathrm{~b}$

00-608 Warsaw

Poland

E-mail: aga.szostek@opi.org.pl
Panos Markopoulos

(Guest Editor)

Department of Industrial Design Eindhoven University of Technology

Den Dolech 2

P.O. Box 513

5600 MB Eindhoven

The Netherlands

E-mail: P.Markopoulos@tue.nl

(C) Szostek and Markopoulos; Licensee Bentham Open.

This is an open access article licensed under the terms of the Creative Commons Attribution Non-Commercial License (http: //creativecommons.org/licenses/ by-nc/3.0/) which permits unrestricted, non-commercial use, distribution and reproduction in any medium, provided the work is properly cited. 